\section{Influence of a sedentary lifestyle on self-reported mental health status among community-dwelling older adults with depression: Implications for secondary prevention efforts}

\author{
Justin B. Dickerson, 1 \\ Matthew Lee Smith,2,3 Marcia G. Ory² \\ 1Department of Health Policy \& \\ Management; 2Department of Social \& \\ Behavioral Health, Texas A\&M Health \\ Science Center, School of Rural Public \\ Health, College Station, Texas; \\ 3Department of Health Promotion \& \\ Behavior, The University of Georgia, \\ College of Public Health, Athens, \\ Georgia, USA
}

\section{Abstract}

The relationship between sedentary behavior and mental health is documented in the primary prevention literature in the context of mental illnesses such as depression. However, these relationships have not been studied to the same extent for secondary prevention efforts, especially among at risk communitydwelling older adults. The Brazos Valley Health Assessment was administered to nearly 4000 adults. Respondents self-reported disease status, lifestyle behaviors such as physical activity and nutrition habits, perceptions of physical and mental health, and socio-demographics. The selection criteria for inclusion in our study sample were being at least 55 years old and having been diagnosed with depression by a healthcare provider. This criterion resulted in a sample of 545 . Negative binomial regression was used to examine the association between number of the past 30 days where mental health days were not good and sedentary behaviors such as time spent watching television and time spent using a computer. For each day per week respondents did not engage in at least 10 min of moderate physical activity (such as fast walking) relative to their peers, they were $5 \%$ less likely to report positive mental health $(\mathrm{IRR}=0.95, \mathrm{P}=0.036)$. For each additional hour per day respondents spent watching television relative to their peers, they were $12 \%$ more likely to report poorer mental health (IRR=1.12, $\mathrm{P}=0.033$ ). Secondary prevention efforts should recognize the influence of sedentary behaviors and emphasize similar interventions used for primary prevention of depression.

\section{Introduction}

Depression is a common medical condition among older community-dwelling adults. Up to $16 \%$ of older adults living independently in the community suffer from clinically significant depression. ${ }^{1}$ This is concerning for many reasons. First, depression exhibits a bi-directional relationship with chronic disease among the older adult population. ${ }^{2}$ This means worsening levels of depression can impair management of existing chronic diseases resulting in poorer physical health and further exacerbating depressive symptoms. Second, depression among older adults can lessen the success of interventions to address negative health behaviors such as tobacco smoking. ${ }^{3}$ Finally, depression can lead to suicidal tendencies that make depression management a key component of secondary prevention efforts for suicide (secondary prevention being defined as efforts to reduce the number of suicide attempts in high-risk populations). ${ }^{4}$

The relationship between sedentary behaviors (e.g. , lack of physical activity and engaging in habits such as time spent watching television and using a computer) and primary prevention of depression (primary prevention being defined as efforts to prevent the occurrence of depression) have been well established in the literature. Garber and colleagues ${ }^{5}$ found community-dwelling older adults with higher scores on the Geriatric Depression Scale (GDS), indicating elevated depression status, also reported poorer levels of physical functioning $(\beta=0.106, \mathrm{P}=0.004)$. However, a recent meta-analysis indicated interventions to improve physical activity can act as primary prevention agents by reducing depressive symptoms before depression is diagnosed. ${ }^{6}$ In particular, aerobic exercise (e.g., moderate physical activity like fast walking) has been shown to be efficacious in reducing depressive symptoms among those of both high and low depressive symptomatologies. ${ }^{7}$ This is likely due to the unique physiological effect of physical activity on depression immunology. ${ }^{8}$ Research has also established that increased sedentary behavior such as screen-based entertainment (e.g., television-watching and entertainment delivered via mediums such as computer screens) is independently associated with poorer mental health status. ${ }^{9}$ Incidentally, similar research also indicates these behaviors are independently associated with a higher risk of mortality despite individuals' level of physical activity. ${ }^{10}$

While many studies of physical activity and depression have been conducted among older adults, most of them have similar characteristics such as cross-sectional selection of adults based solely on demographics such as age, or case-control studies that did not consider the existing
Correspondence: Justin B. Dickerson, Department of Health Policy \& Management, Texas A\&M Health Science Center, School of Rural Public Health, 1266 TAMU, College Station, Texas 77843, USA.

Tel: +1.979.458.0642 - Fax: +1.979.458.4264.

E-mail: dickerson@srph.tamhsc.edu

Key words: sedentary behavior, depression, secondary prevention.

Conflict of interest: the authors report no conflicts of interest.

Contributions: JBD, study design and manuscript writing; MLS, study design, methodology development, results interpretation; MGO, results interpretation, discussion and conclusion sections.

Abstract presentation: an abstract version of this research was presented as a poster presentation at the 2011 Texas Conference on Aging in San Antonio, Texas on June 14, 2011.

Received for publication: 23 June 2011.

Revision received: 30 August 2011.

Accepted for publication: 9 September 2011.

This work is licensed under a Creative Commons Attribution NonCommercial 3.0 License (CC BYNC 3.0).

(C) Copyright J.B. Dickerson et al., 2011

Licensee PAGEPress, Italy

Ageing Research 2011; 3:e6

doi:10.4081/ar.2011.e6

clinical diagnosis of the patients in the study. In essence, these studies have been primary prevention studies aimed at motivating practitioners, policymakers, and researchers to enhance funding and focus on interventions to increase physical activity and reduce sedentary behavior to improve public health outcomes. Although these primary prevention efforts are very important, there have been few studies about sedentary behaviors explicitly focused on communitydwelling older adults who have already been diagnosed with depression by a healthcare provider. It is these clinically diagnosed individuals who are a key focus of suicide-related secondary prevention efforts. They are also individuals who may not experience the same efficacy from interventions to improve mental health status and increase physical activity as witnessed among the primary prevention population. The purposes of this study are to i) examine the influence sedentary behaviors have on selfreported mental health status of communitydwelling older adults who have already been diagnosed with depression by a healthcare provider and ii) determine whether the secondary prevention population requires separate interventions from those currently focused on the primary prevention population. 


\section{Materials and Methods}

\section{Survey instrument}

The Brazos Valley Health Assessment (BVHA) ${ }^{11}$ is conducted every four years by the Center for Community Health Development at the Texas A\&M Health Science Center. The 2010 BVHA was administered using randomdigit dial methodologies to residents of eight counties in central Texas which included the metropolitan statistical area of Bryan College Station. The survey consisted of a 32page instrument comprised of questions from multiple validated sources such as the Centers for Disease Control and Prevention's (CDC) Behavioral Risk Factor Surveillance System (BRFSS). 12 The survey included 3,964 respondents.

\section{Selection criteria}

Respondents to the BVHA were chosen for inclusion in the study sample if they selfreported being at least 55 years of age and also self-reported being diagnosed with depression by a healthcare provider.

\section{Dependent variable}

Mental health days not good was measured by the BVHA in the same manner as the BRFSS. ${ }^{2}$ The variable asked respondents to report how many of the past thirty days their mental health was not good. The variable was measured on a continuous basis from 0 to 30 .

\section{Independent variables}

Socio-demographic variables were used to describe the respondents. These variables were: sex, age, race, and whether the respondent self-reported as Hispanic. Sex was measured as a dichotomous variable (i.e., male or female), age was measured as a continuous variable (i.e., number of years old), race was measured as a categorical variable (i.e., White, African-American, other, or not reported), and self-identification as Hispanic was measured as a dichotomous variable (i.e., yes or no). Variables germane to our research question about the relationship between sedentary behavior and depression were also included. These variables were: days per week spent engaging in at least $10 \mathrm{~min}$ of moderate physical activity (defined in the survey instrument as; your heart beats faster than normal and you can talk but not sing, and accompanied by visual images and text indicating fast walking, strength training and swim gently), hours spent watching television and hours spent using a computer each day for the past month. These variables were measured on a continuous basis from 0 to 7 (days per week spent engaging in at least 10 min of moderate physical activity) and 0 to 24 (hours spent using a computer or watching television per day).

\section{Descriptive statistics}

Both t-statistics (for continuous variables) and chi-square statistics (for categorical variables) were used to detect statistically significant differences in the independent variables of the study by sex. The level of significance was $\alpha=0.05$.

\section{Negative binomial regression with incidence rate ratios}

Given the positive skew inherent in the dependent variable (since the variable was measured on a basis of 0 to 30), Poisson regression and negative binomial regression were evaluated as potential distributions to model the influence of the independent variables on the number of mental health days not good as reported by the respondents. Both distributions were analyzed in Stata version 11 (Stata Corp. LP, College Station, TX, USA). The implicit assumption in the Poisson distribution is that the variance in the data is equal to the mean of the data. In contrast, the negative binomial distribution allows for the estimation of both mean and variance parameters. In reviewing the mean and variance of the dependent variable in our sample, it was determined the variance was nearly twelve times greater than the mean. This provided evidence the negative binomial distribution was the most appropriate method to utilize. We confirmed this belief by evaluating our data against both distributions using the nbvargr function in Stata provided by the UCLA Academic Technology Services, Statistical Consulting Group. ${ }^{13}$ In addition to using negative binomial regression as our method of statistical analysis, we also used corresponding incidence rate ratios to estimate the effect of change in the dependent variable outcome assuming unique changes in the values of independent variables.

\section{Results}

\section{Sample and descriptive statistics}

Table 1 reports the descriptive statistics of the sample by sex. The selection criteria resulted in a sample size of 545 . The sample was comprised mostly of females $(78.2 \%)$. The mean age of the sample was $66.48 \pm 8.87$. The majority of the sample self-reported as White (86.6\%) while $6.4 \%$ self-reported as Hispanic (6.4\%). On average, respondents reported watching television for $3.16 \pm 1.26$ hours per day and using a computer for $2.09 \pm 1.38$ hours

Table 1. Descriptive statistics by sex $(n=545)$.

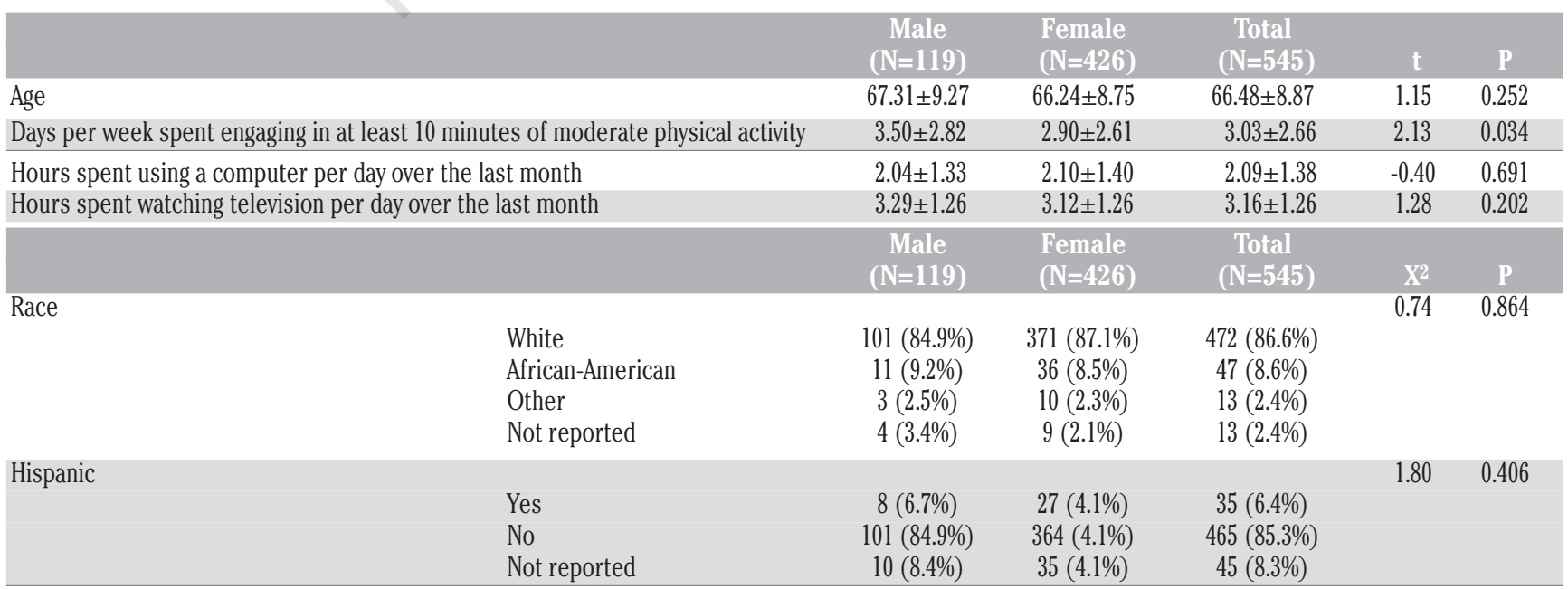


per day. Males were statistically more likely to spend more days per week engaged in at least 10 min of moderate physical activity $(3.50 \pm 2.82)$ than females $(2.90 \pm 2.61)(\mathrm{t}=2.13$, $\mathrm{P}=0.034)$.

\section{Influences on number of mental health days not good}

Table 2 reports the results of the negative binomial regression model with incidence rate ratios. The model fit measures indicated an acceptable risk (less than 5\%) of Type 1 error. The independent variables examined in the descriptive statistics analysis were used as predictors in the model to identify potential influences on the number of mental health days not good as self-reported by the respondents. None of the socio-demographic variables exhibited statistically significant influence on the number of mental health days not good. However, among the sedentary behavior variables, both the number of days per week spent engaging in at least 10 min of moderate physical activity $(\beta=-0.05, P=0.036)$ and the number of hours spent watching television $(\beta=0.11, P=0.033)$ were significantly associated with number of mental health days not good. For every day per week respondents did not engage in at least 10 min of physical activity, they were $5 \%$ less likely to report number of days where mental health was good relative to their peers who exercised more frequently (IRR=0.95, CI = 0.90-0.97). For every additional hour respondents spent watching television per day relative to peers, they were $12 \%$ more likely to report a higher number of days mental health was poor $(\mathrm{IRR}=1.12, \mathrm{CI}=1.01-1.24)$.

\section{Discussion}

\section{Opportunities to engage older adults in physical activity to address depression}

The results of this study indicate sedentary behaviors remain significantly associated with depression after initial diagnosis. As such, it is important to look for community-based opportunities to engage with older adults to address depression and promote healthier lifestyle behaviors. Studies ${ }^{14,15}$ indicate community senior centers provide an accessible environment for older adults that foster opportunities for physical activity and social interaction. Even among older adults living in self-care retirement facilities, physical activity programs show efficacy in reducing depressive symptoms among those already diagnosed with depression. ${ }^{16}$ Additional research indicates that with the proper amount of physical exercise and social support, the setting where older adults receive services becomes less relevant in achieving reduced depressive symptoms. ${ }^{17}$ The key point is older adults need organizations that promote physical activity and provide opportunities for exercise and social interaction. Despite the fact older adults watch more television than their younger counterparts; research suggests this is primarily due to their lack of alternatives rather than their satisfaction with watching television. ${ }^{18}$ If public health practitioners can offer healthier substitutes for sedentary behavior, there is reason to believe older adults will take advantage of such opportunities with positive effects on depressive symptomatology.

\section{Implications for secondary preven-} tion of depression

This study has established the importance of addressing sedentary behaviors among those already diagnosed with depression. These findings have been discussed in the context of secondary prevention for depression such as treating the condition before more serious outcomes such as suicide can occur. However, there are also benefits of taking such action for secondary prevention of other diseases. Mortality rates of patients being treated for acute coronary syndrome events have been shown to be influenced by whether or not the patient is persistently depressed.19,20 This is because depressed patients can often falter in their adherence to post-event treatment regimens such as medication management and lifestyle modifications such as improved diet and exercise. ${ }^{19}$ Similar impaired adherence has been seen for depressed patients being treated for human immunodeficiency virus, diabetes, and other chronic diseases. ${ }^{19}$ Addressing depressive symptoms among older adults already diagnosed with depression not only has positive benefits in addressing secondary prevention of depression, but can also markedly improve secondary prevention of these and other chronic diseases linked to depression.

\section{Limitations}

Focusing on a population of older adults diagnosed with depression is a unique approach in examining the relationship of sedentary behavior and depression. However, the study also has its limitations. First, the data in the study was self-reported making it

Table 2. Negative binomial regression model with incidence rate ratios.

\begin{tabular}{|c|c|c|c|c|c|c|}
\hline \multicolumn{7}{|c|}{ Dependent variable: days where mental health was poor } \\
\hline \multicolumn{7}{|l|}{$\begin{array}{l}\text { Model fit measures } \\
\text { Log likelihood }=-1530.02 \\
\text { LR } \chi^{2}(7)=14.90 \\
\text { Prob }>\chi^{2}=0.037\end{array}$} \\
\hline \multirow[t]{2}{*}{$\begin{array}{l}\text { Independent Variable/ } \\
\text { Measurement }\end{array}$} & \multirow[t]{2}{*}{ Coefficient } & \multirow[t]{2}{*}{ Standard error } & \multirow[t]{2}{*}{$\mathbf{P}$} & \multirow[t]{2}{*}{ Incidence rate ratio } & \multicolumn{2}{|c|}{$\begin{array}{c}\text { Incidence rate ratio } \\
95 \% \text { Confidence interval }\end{array}$} \\
\hline & & & & & Lower & Upper \\
\hline Age (Continuous) & -0.01 & 0.01 & 0.284 & 0.99 & 0.98 & 1.01 \\
\hline Sex $(1=$ Male, 2 = Female $)$ & -0.15 & 0.16 & 0,358 & 0.86 & 0.63 & 1.18 \\
\hline African-American $(1=$ Yes, $0=$ No) & 0.24 & 0.25 & 0.323 & 1.27 & 0.79 & 2.06 \\
\hline Hispanic $(1=$ Yes, 0 = No $)$ & 0.14 & 0.27 & 0.597 & 1.15 & 0.68 & 1.94 \\
\hline $\begin{array}{l}\text { Days per week spent engaging in at least } \\
10 \text { min of moderate physical } \\
\text { activity (Continuous) }\end{array}$ & -0.05 & 0.02 & 0.036 & 0.95 & 0.90 & 0.97 \\
\hline $\begin{array}{l}\text { Hours spent using a computer per day over } \\
\text { the last month (Continuous) }\end{array}$ & -0.04 & 0.05 & 0.359 & 0.96 & 0.87 & 1.05 \\
\hline $\begin{array}{l}\text { Hours spent watching television per day } \\
\text { over the last month (Continuous) }\end{array}$ & 0.11 & 0.05 & 0.033 & 1.12 & 1.01 & 1.24 \\
\hline
\end{tabular}


susceptible to reliability and validity bias. Second, this study took place in a small geographic area which minimizes generalizability of the findings. Third, the depression variable in the study was not measured using a scale such as the GDS. This limited the ability of the researchers to understand the severity of the self-reported depression of the respondents.

\section{Conclusions}

Primary prevention of depression among older adults is a well documented public health need. However, our study illustrates the need to continue these interventions for secondary prevention efforts as well. We have provided evidence of opportunities in the community available to public health practitioners to help overcome the challenges of depressive symptoms among this population. Further, we have identified the health benefits of taking action to address this population, and associated benefits to secondary prevention of other diseases. The challenge left for practitioners is to take this knowledge and ensure physical activity programs used for primary prevention of depression are also considered for secondary prevention of depression and other diseases.

\section{References}

1. Blazer D. Depression in Late Life: Review and Commentary. J Gerontol A Biol Sci Med Sci 2003;58A:249-65.

2. Fiest KM, Currie SR, Williams JVA, Wang J. Chronic conditions and major depression in community-dwelling older adults. J Affect Disord 2011;131:172-8.

3. Kenney BA, Holahan CJ, Holahan CK, et al. Depressive symptoms, drinking problems, and smoking cessation in older smokers.
Addict Behav 2009;34:548-53.

4. Ganz D, Braquehais MD, Sher L. Secondary Prevention of Suicide. PLoS Medicine 2010;7:1-4.

5. Garber CE, Greaney ML, Riebe D, et al. Physical and mental health-related correlates of physical function in community dwelling older adults: a cross sectional study. BMC Geriatr 2010;10:6.

6. Conn VS. Depressive symptom outcomes of physical activity interventions: metaanalysis findings. Ann Behav Med 2010; 39:128-38.

7. Penninx B, Rejeski WJ, Pandya J, et al. Exercise and depressive symptoms: a comparison of aerobic and resistance exercise effects on emotional and physical function in older persons with high and low depressive symptomatology. J Gerontol B Psychol Sci Soc Sci 2002;57B:P124-32.

8. Landi F, Abbatecola AM, Provinciali M, et al. Moving against frailty: does physical activity matter? Biogerontology 2010;11: 537-45.

9. Hamer M, Stamatakis E, Mishra GD. Television- and Screen-Based Activity and Mental Well-Being in Adults. Am J Prev Med 2010;38:375-80.

10. Stamatakis E, Hamer M, Dunstan DW. Screen-Based Entertainment Time, AllCause Mortality, and Cardiovascular Events: Population-Based Study With Ongoing Mortality and Hospital Events Follow-Up. J Am Coll Cardiol 2011;57:2929.

11. Center for Community Health Development, Texas A\&M Health Science Center, School of Rural Public Health. Brazos Valley Health Assessment Executive Report. 2010. Available from: http://www.tamhsc.edu/about/accreditation/sacs/3-4/3-4-2/Regional_Health_ Assessment_Report.pdf

12. Centers for Disease Control and Prevention. Measuring healthy days:
Population assessment of health-related quality of life. 2000. Available from: http:/www.cdc.gov/hrqol/pdfs/mhd.pdf

13. UCLA: Academic Technology Services, Statistical Consulting Group. Stata Library: Analyzing Count Data. 2011. http:/www.ats.ucla.edu/stat/stata/library/c ount.htm Accessed: 6 July 2011.

14. Fulbright SA. Rates of depression and participation in senior centre activities in community-dwelling older persons. J Psychiatr Ment Health Nurs 2010;17:38591.

15. Hickerson B, Moore A, Oakleaf L, et al. The Role of a Senior Center in Promoting Physical Activity for Older Adults. J Park Recreat Admin 2008;26:22-39.

16. Brown AK, Liu-Ambrose T, Tate R, Lord SR. The effect of group-based exercise on cognitive performance and mood in seniors residing in intermediate care and self-care retirement facilities: a randomised controlled trial. Br J Sports Med 2009;43:60814.

17. Choi NG, McDougall GJ. Comparison of depressive symptoms between homebound older adults and ambulatory older adults. Aging Ment Health 2007;11:310-22.

18. Depp CA, Schkade DA, Thompson WK, Jeste DV. Age, Affective Experience, and Television Use. Am J Prev Med 2010;39: 173-8.

19. Kronish IM, Rieckmann N, Halm EA, et al. Persistent Depression Affects Adherence to Secondary Prevention Behaviors After Acute Coronary Sybdromes. J Gen Intern Med 2006;21:1178-83.

20. McGee HM, Doyle F, Conroy RM, et al. Impact of briefly-assessed depression on secondary prevention outcomes after acute coronary syndrome: a one-year longitudinal survey. BMC Health Serv Res 2006;6:9-8. 